\title{
SURREAL NUMBERS WITH DERIVATION, HARDY FIELDS AND TRANSSERIES: A SURVEY
}

\author{
VINCENZO MANTOVA AND MICKAËL MATUSINSKI
}

To the memory of Murray Marshall.

\begin{abstract}
The present article surveys surreal numbers with an informal approach, from their very first definition to their structure of universal real closed analytic and exponential field. Then we proceed to give an overview of the recent achievements on equipping them with a derivation, which is done by proving that surreal numbers can be seen as transseries and by finding the 'simplest' structure of H-field, the abstract version of a Hardy field. All the latter notions and their context are also addressed, as well as the universality of the resulting structure for surreal numbers.
\end{abstract}

\section{INTRODUCTION}

The theory of surreal numbers initiated by J.H. Conway in [Con76], and popularized by D. Knuth [Knu74], is fascinating and fruitful but not so well known. However, it has been enhanced continuously and now, several remarkable achievements have been reached.

Rooted into very fundamental and accessible set theoretic considerations, these objects nonetheless strike by the richness together with the universality of their structure. As a class of numbers - denote it by No-they include simultaneously the real numbers and the ordinal numbers, unified into a common algebraic structure: a huge real closed field. Moreover, No can be viewed as a field of (generalized) power series with real coefficients, being accordingly a - one can even say "the" - universal domain for all real closed fields. Moreover, after [Gon86], No carries exponential and logarithmic functions.

These remarkable facts resonated with important results in differential equations [É92] and model theory [Wi196, vdDMM94] concerning tame geometry (nonoscillating real functions and their formal analogues). Several similar big real closed fields of formal power series with exponential and logarithm - called transseries, or log-exp series, or exp-log series - have been developed, but with an important additional item: a well-behaved derivation. In fact, the purpose of these fields is precisely to build algebraic structures being closed under resolution of differential equations in terms of formal analogues of non-oscillating real functions. This purpose appears to have reached a milestone recently with the quantifier elimination result in [ADH1].

Related to this, one of the recent achievements concerning surreal numbers is the construction in [BM] of a well-behaved derivation in No. Moreover, for this derivation No is closed under integration and even the results in [ADH1] apply: No can be said to be "the" universal domain for non-oscillating differentiable real functions according to [ADH2]. 
The present survey article is divided into three sections and has two aims: provide a gentle and intuitive introduction to surreal numbers and give an overview of these new results about derivation within their context. In particular, note that we won't address several other old and new interesting results, e.g. the ones about analysis on surreal numbers like [CEF] or the ones about number theory through the notion of integer part like [EK]. To survey these results would lead us way further and to a much longer article. We will try to stay guided by the following nice fact. As mathematical objects, surreal numbers can be viewed in three different manners: as numbers in the set theoretic sense (cuts between ordered sets: see Definition 2.1), as combinatorial objects (ordered binary sequences: see Definition 2.2) or as formal analogues of non-oscillating real functions (generalized power series: see Theorem 2.10). This ubiquity can explain the possibility for surreal numbers to be used into different contexts, in particular as an ordered algebraic structure from the model theoretic and tame geometric point of view. However, we will be more interested in the third point of view and will provide in the second section a survey on the corresponding functional and formal objects (Hardy fields, transseries, H-fields). The last section will be devoted to survey the results in [BM, ADH2] and their corollaries.

The authors would like to thank A. Gehret, L. van den Dries and an anonymous referee for the useful comments and corrections.

\section{Surreal Numbers}

2.1. Surreal numbers consist of "all numbers great and small". The construction of surreal numbers goes back to [Con76]. One of the most striking ideas of J.H. Conway is to merge two fundamental constructions of numbers: Dedekind's construction of real numbers in terms of cuts in the set of rational numbers, and von Neumann's construction of ordinal numbers by transfinite induction in terms of set membership. All that is needed to get started with surreal numbers is the basis of set theory (to be precise: the axiomatic "NEG with Global Choice", which extends conservatively "ZFC", see e.g. [FS96]), the following two definitions and nothing more than the empty set $\emptyset$. Let us paraphrase J.H. Conway:

Definition 2.1 (Conway).

(S1) If $L, R$ are any two sets of numbers, and no member of $L$ is $\geq$ any member of $R$, then there is a number $\{L \mid R\}$. All numbers are constructed in this way.

(S2) For two numbers $a=\{L \mid R\}$ and $b=\left\{L^{\prime} \mid R^{\prime}\right\}$, we say that $a \geq b$ iff no member of $R$ is $\leq b$ and no member of $L^{\prime}$ is $\geq a$. Note that $a \leq b$ just means $b \geq a$.

(S3) For two numbers $a, b$, we say that $a=b$ if $a \leq b$ and $b \leq a$.

The very first number has to be $\{\emptyset \mid \emptyset\}$ which is naturally called the number 0 . Then come $1:=\{0 \mid \emptyset\}$ and $-1:=\{\emptyset \mid 0\}$. We leave it to the reader to check that these 3 pairs actually define surreal numbers, and that they verify: $-1<0<1$. Likewise, one obtains at the next step for example:

$$
-2:=\{\emptyset \mid-1\}<-1<-\frac{1}{2}:=\{-1 \mid 0\}<0<\frac{1}{2}:=\{0 \mid 1\}<1<2:=\{1 \mid \emptyset\} .
$$


Actually, just by considering numbers of the type $\{L \mid \emptyset\}$ and by transfinite induction, we can construct any well-ordered set. E.g., all the natural numbers are recovered via the simple induction formula: $n+1:=\{n \mid \emptyset\}$, and the first infinite ordinal is:

$$
\omega:=\{1,2,3, \ldots \mid \emptyset\} .
$$

In other words, the proper class of ordinal numbers - denote it by $\mathbf{O n}$ - is included into surreal numbers, the proper class of the latter being denoted by No. Likewise, anti-well-ordered sets are defined by restricting to the numbers $\{\emptyset \mid R\}$. The choice of $1 / 2$ and $-1 / 2$ for the preceding surreal numbers, with its implicit arithmetical content, will be justified thereafter.

Due to their inductive construction, surreal numbers naturally form a hierarchy. More precisely, to each surreal number one can assign its birthday: the ordinal number at which it has been constructed [Con76, Theorem 16, p.30]. Moreover, any surreal number $a$ of birthday $\alpha$ is equal to a cut $\{L \mid R\}$ among the numbers with birthday less than $\alpha$. Therefore, for any ordinal $\beta<\alpha$, $a$ defines a cut among the numbers with birthday less than $\beta$ :

$$
\{b<a, \operatorname{birthday}(b)<\beta \mid b>a, \operatorname{birthday}(b)<\beta\},
$$

the latter cut being itself a number $a_{\beta}$ of birthday $\beta$. Following P. Ehrlich's terminology [Ehr94], such $a_{\beta}$ is said to be simpler than $a$, denoted by $a_{\beta}<_{s} a$. The relation $\leq_{s}$ is a well-founded partial order relation on No. Conway comes to this observation after establishing that $\mathbf{N o}$ can be viewed as a lexicographically ordered binary tree [Con76, p.11] (see Figure 1).

Then, with the preceding notations, the $a_{\beta}$ 's are exactly the nodes above $a$, and $a$ itself as a node gives rise at the next stage to exactly two new surreal numbers, one at its immediate left and another at its immediate right. Now, to each branch of the tree a sign can be attributed $\ominus$ if the branch tilts to the left, $\oplus$ if it tilts to the right. This leads us to another representation for a surreal number: the sequence of signs which expresses the unique path connecting it to the common root 0 . We call it the sign sequence representation of a surreal number. Conversely, any sign sequence of length some ordinal is the sign sequence of a unique surreal number [Con76, Theorem 18].

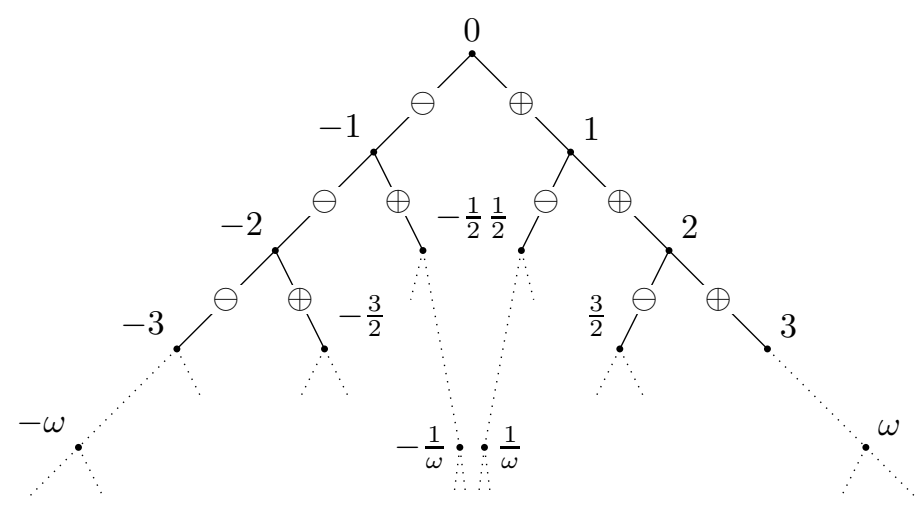

Figure 1. The tree of surreal numbers. 
Gonshor takes the existence of such a representation as a starting point for the theory of No, assuming that the proper class On of ordinal numbers is given. In [Gon86], he uses the following equivalent definition for No:

Definition 2.2 (Gonshor). A surreal number is any function from a given ordinal number to the set of two elements: $\{\ominus, \oplus\}$, i.e. informally, a sequence of pluses and minuses indexed by some ordinal.

The ordinal $\alpha$ on which the surreal number is defined is called its length - set $l(a):=\alpha-$ which coincides with the birthday of $a$. The partial ordering on No called the simplicity ordering is defined as:

$a \leq_{s} b \Leftrightarrow$ the sign sequence of $a$ is an initial subsequence of that of $b$.

For the total ordering, let $a$ and $b$ be any two surreal numbers with $l(a) \leq$ $l(b)$. Consider the sign sequence of a completed with 0's so that the two sign sequences have same length. Then consider the lexicographical order between them, denoted by $\leq$, based on the following relation:

$$
\ominus<0<\oplus
$$

e.g. the inequalities (2.1) can be written as:

$$
\ominus \ominus<\ominus<\ominus \oplus<0<\oplus \ominus<\oplus<\oplus \oplus \text {. }
$$

Returning to Conway's construction, the reader might have noticed a seeming ambiguity. What is for instance the number $\{-1 \mid 1\}$ ? Or $\{-2 \mid 1 / 2,1,2\}$ ? In fact, both of them are equal to 0 , i.e. $\{\emptyset \mid \emptyset\}$. This indicates that the representation of a number as an actual cut $\{L \mid R\}$ is not unique. The following notion appearing in Gonshor's book [Gon86, p.9] helps to clarify the situation a bit. Take two pairs $(L, R)$ and $\left(L^{\prime}, R^{\prime}\right)$ of subsets of No with $L<R$ and $L^{\prime}<R^{\prime} .\left(L^{\prime}, R^{\prime}\right)$ is said to be cofinal in $(L, R)$ if for any $(a, b) \in L \times R$, there is $\left(a^{\prime}, b^{\prime}\right) \in L^{\prime} \times R^{\prime}$, such that $a \leq a^{\prime}<b^{\prime} \leq b$. $\left(L^{\prime}, R^{\prime}\right)$ and $(L, R)$ are mutually cofinal if $\left(L^{\prime}, R^{\prime}\right)$ is cofinal in $(L, R)$ and $(L, R)$ is cofinal in $\left(L^{\prime}, R^{\prime}\right)$. One has that:

Theorem 2.3 (Cofinality theorem [Gon86, Thms. 2.6-2.7]). Let $a=\{L \mid R\}$ and $\left(L^{\prime}, R^{\prime}\right)$ be cofinal in $(L, R)$. Then:

- if $L^{\prime}<a<R^{\prime}$, then $a=\left\{L^{\prime} \mid R^{\prime}\right\}$;

- if $(L, R)$ is cofinal in $\left(L^{\prime}, R^{\prime}\right)$, namely if $(L, R)$ and $\left(L^{\prime}, R^{\prime}\right)$ are mutually cofinal, then $a=\left\{L^{\prime} \mid R^{\prime}\right\}$.

Theorem 2.4 (Inverse cofinality theorem [Gon86, Thms. 2.8-2.9]). Let a be $a$ number, and let $L_{a}:=\left\{b \in \mathbf{N o} ; b<a\right.$ and $\left.b<_{s} a\right\}, R_{a}:=\{b \in \mathbf{N o} ; b>$ $a$ and $\left.b<_{s} a\right\}$. Then $a=\left\{L_{a} \mid R_{a}\right\}$. Moreover, if $a=\left\{L^{\prime} \mid R^{\prime}\right\}$, then $\left(L^{\prime}, R^{\prime}\right)$ is cofinal in $\left(L_{a}, R_{a}\right)$.

The simplest surreal number that lies between -1 and 1 , or between -2 and $\{1 / 2,1,2\}$, is indeed 0 . For any surreal number $a$, its representation $\left\{L_{a} \mid R_{a}\right\}$ for $L_{a}$ and $R_{a}$ as above is called the canonical cut of $a$. By abuse of notation, we also denote the canonical cut by $a=\left\{a^{L} \mid a^{R}\right\}$ where $a^{L}$ and $a^{R}$ are general elements of the canonical sets $L_{a}$ and $R_{a}$ (e.g. $a^{L}=n$ if $L_{a}=\mathbb{N}$ ).

Now the notations $1 / 2:=\{0 \mid 1\}$ and $-1 / 2:=\{1 \mid 0\}$ can be partly justified by the following intuitive statement: they are the simplest numbers between 0 and 1 , and -1 and 0 respectively. In fact, any surreal number of finite length corresponds to a dyadic fraction. Subsequently, among the surreals of length $\omega$, we find the rationals 
non-dyadic and the irrational real numbers. Note that we also get a priori non-real numbers, like the simplest infinitely large number $\omega$ (recall that $\omega:=\{n \mid \emptyset\}$ ), or the simplest infinitesimal one $\left\{0 \mid 1 / 2^{n}\right\}$ to which we assign the symbol $1 / \omega$. In the following section such an assignation will be plainly justified from an algebraic standpoint.

2.2. A universal domain for real closed fields. The next tour de force in Conway's construction is not only to reconstruct numbers as ordered elements but also to recover the algebraic relations between them, and even to uncover some new ones.

Definition 2.5 (Field operations). For any surreal numbers $a=\left\{a^{L} \mid a^{R}\right\}$ and $b=\left\{b^{L} \mid b^{R}\right\}$, let us define:

$$
\begin{array}{lccl}
\text { addition: } & a+b:= & \left\{a^{L}+b, a+b^{L} \mid a^{R}+b, a+b^{R}\right\} \\
\text { inverse element: } & -a & := & \left\{-a^{R} \mid-a^{L}\right\} ; \\
\text { neutral element: } & 0 \quad= & \{\emptyset \mid \emptyset\} ; \\
\text { multiplication: } & a \cdot b:= & \left\{a^{L} \cdot b+a \cdot b^{L}-a^{L} \cdot b^{L},\right. \\
& & a^{R} \cdot b+a \cdot b^{R}-a^{R} \cdot b^{R} \mid \\
& & a^{L} \cdot b+a \cdot b^{R}-a^{L} \cdot b^{R}, \\
& & \left.a^{R} \cdot b+a \cdot b^{L}-a^{R} \cdot b^{L}\right\} \\
& & & \{0 \mid \emptyset\}=\oplus .
\end{array}
$$

First of all, we need to check that these inductive formulas are well-defined. For the definitions of the addition and of the inverse element, one can observe that the elements of the left hand set of the cut formula are $<$ the elements of the right hand set, for instance $a+b^{L}<a^{R}+b$. For the multiplication, the corresponding necessary condition is less obvious. It relies on the following type of observations:

$$
\begin{aligned}
\left(a-a^{R}\right)\left(b-b^{L}\right)<0< & \left(a-a^{L}\right) \cdot\left(b-b^{L}\right) \Leftrightarrow \\
& a^{L} \cdot b+a \cdot b^{L}-a^{L} \cdot b^{L}<a \cdot b<a^{R} \cdot b+a \cdot b^{L}-a^{R} \cdot b^{L} .
\end{aligned}
$$

Let us illustrate by an example the multiplication formula. Consider $\omega:=\{n \mid \emptyset\}$ and $1 / \omega:=\left\{0 \mid 1 / 2^{m}\right\}$ (recall that $n$ and $m$ implicitly denote arbitrary positive integers) and suppose that we have already defined the multiplication of simpler pairs of numbers. Then:

$$
\begin{aligned}
\omega \cdot \frac{1}{\omega} & =\{n \mid \emptyset\} \cdot\left\{0 \mid \frac{1}{2^{m}}\right\} \\
& =\left\{n \cdot \frac{1}{\omega}+0 \cdot \omega-0 \cdot n \mid n \cdot \frac{1}{\omega}+\omega \cdot \frac{1}{2^{m}}-n \cdot \frac{1}{2^{m}}\right\} \\
& =\left\{n \cdot \frac{1}{\omega} \mid n \cdot \frac{1}{\omega}+(\omega-n) \cdot \frac{1}{2^{m}}\right\} \\
& =1 \quad \text { since } n \cdot \frac{1}{\omega}<1<(\omega-n) \cdot \frac{1}{2^{m}} .
\end{aligned}
$$

There is no simple formula in terms of sign sequences for the various operations, except for the additive inverse of a surreal number: the sign sequence of $-a$ is derived from that of $a$ by changing $\oplus$ 's into $\ominus$ 's and $\ominus$ 's into $\oplus$ 's.

Note that Gonshor proves the so-called uniformity properties for these operations as well as for the maps that will be defined later on [Gon86, Theorems 3.2, 3.5 etc.]. For example in the case of the addition, this means that $a+b$ may be obtained 
by taking any cuts $a=\{L \mid R\}$ and $b=\left\{L^{\prime} \mid R^{\prime}\right\}$ in the respective cofinality classes instead of taking their canonical cuts, and applying the same formula as above. In other words, the formulas do not depend on the cuts for which $a^{L}, a^{R}, b^{L}, b^{R}$ can be taken as general elements.

We already identified real numbers in terms of cuts among the dyadic fractions:

Definition 2.6. $a$ is a real number iff:

$$
a=\left\{a-\frac{1}{2^{n}} \mid a+\frac{1}{2^{n}}\right\}
$$

(where it is understood that $n$ varies in $\mathbb{N}$ ).

In terms of sign sequences, Gonshor establishes that real numbers are those with either finitely many signs or with length $\omega$ and not ultimately of constant sign. On real numbers, the previously defined operations restrict well and one thereby obtains a subfield of No having the least upper bound property, i.e. a copy of the field $\mathbb{R}$.

Addition and multiplication also restrict well to ordinal numbers: they correspond to the notions of natural sum and product, also called Hessenberg operations. Recall that these operations consist in taking the Cantor normal form of ordinal numbers and applying addition and multiplication on them as if they were actual polynomials in an abstract variable $\omega$.

Following Conway, a proper class with a field structure is called a Field. Concerning No itself, one obtains:

Theorem 2.7 ([Con76, Ch. 1], [Gon86, Theorems 3.3, 3.6, 3.7 ]). The class No endowed with its ordering $\leq$ and the operations,+- and $\cdot$ is a totally ordered Field which contains $\mathbb{R}$ and On.

No being an ordered Field carries a natural valuation (see e.g. [PC83, Ch. II, Sect. 4, Satz 1] or [Kuh00, p.16]) which sends an element to the class of elements that are Archimedean equivalent - write $a \asymp b$ - to itself, i.e. for any $a \in$ No, denoting its absolute value by $|a|=\max \{a,-a\}$ :

$$
[a]:=\{b \in \mathbf{N o}|\exists n \in \mathbb{N},| a|\leq n \cdot| b \mid \text { and }|b| \geq n \cdot|a|\} .
$$

Conway says that $a$ and $b$ are commensurate, whereas Gonshor says that $a$ and $b$ have same order of magnitude. Note that the positive part of such equivalence class is always convex, so it needs to have a unique element of minimal length. In other words, there is a canonical complete system of representatives of the Archimedean equivalence classes which forms a cross section of the value group. Conway finds a way to express naturally such elements as the images of his so-called $\omega-\mathbf{m a p}$, a map that generalizes the classical ordinal exponentiation:

Theorem 2.8 ([Con76, Ch.3, Theorems 19 and 20], [Gon86, Theorems 5.1 to 5.4]). The recursive formula:

$$
\forall a \in \mathbf{N o}, \quad \omega^{a}:=\left\{0, n \cdot \omega^{a^{L}} \mid \omega^{a^{R}} / 2^{n}\right\}
$$

(where it is understood that $n$ varies in $\mathbb{N}$ ) defines an ordered Group morphism:

$$
\begin{aligned}
\Omega:(\mathbf{N o},+,<) & \rightarrow\left(\mathbf{N o}_{>0}, \cdot,<\right) \\
a & \mapsto \Omega(a):=\omega^{a}
\end{aligned}
$$


that extends the exponentiation with base $\omega$ of the ordinals. Moreover, for any $a \in \mathbf{N o}$, the surreal number $\omega^{a}$ is the positive representative of minimal length in its own Archimedean equivalence class.

Denote by $\omega^{\text {No }}$ the Image of the $\omega$-map. Note that the residue field $\mathbb{R}$ of the natural valuation is naturally embedded in No. Thus, one has an approximation algorithm: for any surreal number $a$, there are a surreal number $b$ and a real number $r$ such that $\left|a-\omega^{b} \cdot r\right|$ is less than $|a|$ and $\left|\omega^{b} \cdot r\right|$ and in a different Archimedean class, which we denote as $a-\omega^{b} \cdot r \prec a$ and $a-\omega^{b} \cdot r \prec \omega^{b}$, and also as $a \sim \omega^{b} \cdot r$. Such $b$ is called $\operatorname{Ind}(a)$ in [Gon86, Ch.10, Sect.C], defining thus a map which is an incarnation of the natural valuation in the sense of Krull. The value group is (isomorphic to) No viewed as an ordered additive Group, whose multiplicative copy is the cross section $\omega^{\mathrm{No}}$. We are in the situation where an adapted version of Kaplansky's Embedding Theorem (see [Kap42][PC83, Satz 21, p.62]) applies. We can say that No is weakly spherically complete, namely every set of balls directed by inclusion has non-empty intersection. Equivalently, any pseudo-Cauchy sequence indexed by some ordinal has a pseudo limit in No. Subsequently, No is isomorphic to the following Field of formal power series $\mathbb{R}\left(\left(\omega^{\mathbf{N o}}\right)\right)$, i.e. the class of formal expressions $\sum_{i<\lambda} \omega^{a_{i}} \cdot r_{i}$ where $\lambda \in \mathbf{O n},\left(a_{i}\right)_{i<\lambda}$ is a strictly decreasing sequence in No and for any $i<\lambda, r_{i} \in \mathbb{R} \backslash\{0\}$.

Remark 2.9. Note that we are abusing the notation $\mathbb{R}\left(\left(\omega^{\mathbf{N o}}\right)\right)$, since it usually denotes the full Field of generalized power series (also called Hahn series field or Malcev-Neumann series field: see e.g. [Mat14]) which is bigger than No. Indeed, in this context, the field of generalized series, as a maximally valued field, should consist of series with supports being classes. This corresponds to pseudo-Cauchy sequences indexed by classes.

Conway and Gonshor respectively establish two direct constructive proofs of the description of surreal numbers as power series. They are based on the following key fact: one does have in No a notion of convergence for such generalized series. Indeed, any generalized series $\sum_{i<\lambda} \omega^{a_{i}} \cdot r_{i}$ is defined in No as the simplest surreal number having exactly such expansion. In other words, if $\lambda=\alpha+1$, then $\sum_{i<\lambda} \omega^{a_{i}}$. $r_{i}:=\sum_{i<\alpha} \omega^{a_{i}} \cdot r_{i}+\omega^{\lambda} \cdot r_{\lambda}$. If $\lambda$ is a limit ordinal, then $\sum_{i<\lambda} \omega^{a_{i}} \cdot r_{i}$ is defined as the cut obtained as follows for $\epsilon \in \mathbb{R}_{>0}$ :

$$
\begin{aligned}
& \sum_{i<\lambda} \omega^{a_{i}} \cdot r_{i}:= \\
& \qquad\left\{\sum_{i \leq \alpha} \omega^{a_{i}} \cdot r_{i}+\omega^{a_{\alpha}} \cdot\left(r_{\alpha}-\epsilon\right), \alpha<\lambda \mid \sum_{i \leq \alpha} \omega^{a_{i}} \cdot r_{i}+\omega^{a_{\alpha}} \cdot\left(r_{\alpha}+\epsilon\right), \alpha<\lambda\right\} .
\end{aligned}
$$

Consequently, the approximation algorithm leads to a unique expansion of a surreal number as a generalized series. Indeed, if the expansion of a surreal number a were indexed over all the ordinals, then its length $l(a)$ would be greater than On. Thus we obtain an actual new expression for surreal numbers:

Theorem 2.10 (Conway normal form of surreal numbers [Gon86, Theorems 5.5 to 5.8]). Any surreal number $a \in \mathbf{N o}$ can be written uniquely as

$$
a=\sum_{i<\lambda} \omega^{a_{i}} \cdot r_{i}, \quad \text { the normal form of } a,
$$


where $\lambda \in \mathbf{O n}$, the transfinite sequence $\left(a_{i}\right)_{i<\lambda}$ is strictly decreasing and for any $i<\lambda, r_{i} \in \mathbb{R} \backslash\{0\}$. Thus:

$$
\mathbf{N o}=\mathbb{R}\left(\left(\omega^{\mathbf{N o}}\right)\right) .
$$

where $\omega^{\text {No }}$ is seen as the Group of (generalized) monomials.

We recall that the addition of such generalized series is termwise and the multiplication is (the straightforward generalization of) the convolution product for power series (Cauchy product). For ordinal numbers, the Conway normal form coincides with the classical Cantor normal form.

Example 2.11. For any non-negative integers $n$ and $m$, one has that:

$$
\omega^{-n}=\left\{0 \mid \frac{\omega^{-(n-1)}}{2^{k}}\right\},
$$

and in particular:

$$
\omega^{0}:=\{0 \mid \emptyset\}=1, \quad \omega^{-1}=\left\{0 \mid \frac{1}{2^{k}}\right\}=\frac{1}{\omega}(k \in \mathbb{N}) .
$$

Moreover, by induction on $n+m$ and applying Theorem 2.3:

$$
\begin{aligned}
\omega^{-n} \cdot \omega^{-m}= & \left\{0 \mid \frac{\omega^{-(n-1)}}{2^{k}}\right\} \cdot\left\{0 \mid \frac{\omega^{-(m-1)}}{2^{l}}\right\} \\
= & \left\{0, \frac{\omega^{-(m-1)}}{2^{l}} \cdot \omega^{-m}+\frac{\omega^{-(n-1)}}{2^{k}} \cdot \omega^{-m}-\frac{\omega^{-(n-1)}}{2^{k}} \cdot \frac{\omega^{-(m-1)}}{2^{l}} \mid\right. \\
& \left.\frac{\omega^{-(n-1)}}{2^{k}} \cdot \omega^{-m}, \omega^{-n} \cdot \frac{\omega^{-(m-1)}}{2^{l}}\right\} \\
= & \left\{0 \mid \frac{\omega^{-(m+n-1)}}{2^{i}}\right\} \\
= & \omega^{-(n+m)} .
\end{aligned}
$$

Subsequently, we can compute:

$$
\begin{aligned}
\sum_{n \geq 0} \omega^{-n} & =\left\{\sum_{n=0}^{N} \omega^{-n}-\omega^{-N} \mid \sum_{n=0}^{N} \omega^{-n}+\omega^{-N}\right\}(N \in \mathbb{N}) \\
& =\left\{\frac{1-\omega^{-N-1}}{1-\omega^{-1}}-\omega^{-N} \mid \frac{1-\omega^{-N-1}}{1-\omega^{-1}}+\omega^{-N}\right\} \\
& =\frac{1}{1-\omega^{-1}} \cdot\left\{1-\omega^{-N} \mid 1+\omega^{-N}-2 \cdot \omega^{-N-1}\right\} \\
& =\frac{1}{1-\omega^{-1}} \cdot 1 \\
& =\frac{1}{1-\omega^{-1}} .
\end{aligned}
$$

Remark 2.12. The notion of generalized series goes back to the seminal paper [Hah07] of H. Hahn. There, he uses such construction of formal power series with real coefficients and exponents in an arbitrary ordered set, to prove what is called now the Hahn embedding theorem for ordered Abelian groups. The key tool to obtain such an embedding is the Archimedean equivalence relation. Hence, the Conway normal form of a surreal number can be viewed also as an extension of 
Hahn's result to No viewed as an ordered Abelian Group. Now, considering its multiplicative version $\omega^{\text {No }}$, we can introduce the following natural formalism:

$$
\omega^{a}=\omega^{\sum_{i<\lambda} \omega^{a_{i}} \cdot r_{i}}=: \prod_{i<\lambda}\left(\omega^{\omega^{a_{i}}}\right)^{r_{i}} .
$$

Note that the numbers $\omega^{\omega^{b}}$ for $b \in$ No consists of a canonical complete system of representatives of the equivalence classes of surreal numbers that are multiplicatively equivalent in the following sense: for $a \in \mathbf{N o}_{>0},|a| \succ 1$,

$$
[a]_{\text {mult }}=\left[a^{-1}\right]_{\text {mult }}:=\left\{\left.b^{ \pm 1} \in \mathbf{N o}|\exists n \in \mathbb{N},| a\right|^{n} \geq|b| \text { and }|b|^{n} \geq|a|\right\} .
$$

Note that the Conway normal form of any surreal number $a$ can be split into three parts (each of which possibly trivial) in the following two manners. Firstly:

$$
\begin{aligned}
a & =\sum_{a_{i}>0} \omega^{a_{i}} \cdot r_{i}+r_{i_{0}}+\sum_{a_{i}<0} \omega^{a_{i}} \cdot r_{i} \\
& =\text { purely infinite part }+ \text { constant term }+ \text { infinitesimal part. }
\end{aligned}
$$

Secondly:

$$
\begin{aligned}
a & =\omega^{a_{1}} \cdot r_{1} \cdot(1+\epsilon) \\
& =\text { leading monomial } \cdot \text { leading coefficient } \cdot \text { unit. }
\end{aligned}
$$

For the sake of notation, let $\mathbb{J} \subseteq$ No be the (non-unital) ring of purely infinite surreal numbers.

It follows from the representation of a surreal number as a generalized power series (with real closed residue field $\mathbb{R}$ and divisible valued group No) and from Kaplansky's Embedding Theorem that the Field No is a universal domain for real closed fields in the following strong sense. A subset $S$ of No is said to be initial if it verifies:

$$
\forall(a, b) \in S \times \mathbf{N o}, \quad b<_{s} a \Rightarrow b \in S .
$$

Theorem 2.13 (Universal real closed Field).

(1) [Con76, Ch. 5] [Gon86, Ch. 5, Sect. D] The proper class No is a real closed Field.

(2) [Con76, Theorems 28 and 29] [Ehr01, Theorems 9 and 19] Any divisible ordered Abelian group, respectively any real closed field, is isomorphic to an initial subgroup of $(\mathbf{N o},+)$, respectively an initial subfield of $(\mathbf{N o},+, \cdot)$.

E.g., a surreal number $a=\omega^{a_{1}} \cdot r_{1} \cdot(1+\epsilon)$ has an $n$th root if and only if its leading coefficient $r_{1} \in \mathbb{R}$ has an $n$th root, via the following formula:

$$
a^{1 / n}=\omega^{a_{1} / n} \cdot r_{1}^{1 / n} \cdot(1+\epsilon)^{1 / n}
$$

where $(1+\epsilon)^{1 / n}$ is construed as the corresponding series expansion in powers of $\epsilon$ (straightforward generalization of the Maclaurin-Taylor series expansion for the function $(1+x)^{1 / n}$ of a real variable $\left.x\right)$.

Remark 2.14. The previous comment as well as Example 2.11 provide a glimpse of the following fact [Con76, Remark p.43]: any function analytic in some nonempty domain of the real plane can be extended to the surreal numbers enclosed in such domain via its local power series expansion. This will also be illustrated in the following section. In fact, there is an actual theory of analytic functions over surreal numbers [All87]. 
2.3. Exponential and logarithmic functions. Building on unpublished ideas of Kruskal, Gonshor defines inductively in [Gon86] a surjective exponential map:

$$
\exp :(\mathbf{N o},+,<) \rightarrow(\mathbf{N o}>0, \cdot,<)
$$

by means of the power series expansion of the real exponential map:

$$
e^{x}=\sum_{k \geq 0} \frac{x^{k}}{k !}
$$

He obtains also a partial inductive definition of its reciprocal function, the logarithmic map $\log :\left(\mathbf{N o}_{>0}, \cdot\right) \rightarrow(\mathbf{N o},+)$. This terminology is also justified by the following key fact: these maps coincide with the usual exponential and logarithm on real numbers, and with (the extension to surreal numbers of) the MaclaurinTaylor series (2.4) in powers of $a$ for any infinitesimal surreal $a$. Let us denote $E_{n}(x):=\sum_{k=0}^{n} \frac{x^{k}}{k !}$.

Theorem 2.15 ([Gon86, Theorems 10.1 to 10.9, Corollaries 10.1 to 10.3]). Consider the recursive formula:

$$
\begin{gathered}
\forall a \in \mathbf{N o}, \exp (a):= \\
\left\{0, \exp \left(a^{L}\right) E_{n}\left(a-a^{L}\right), \exp \left(a^{R}\right) E_{2 n+1}\left(a-a^{R}\right) \mid \frac{\exp \left(a^{R}\right)}{E_{n}\left(a^{R}-a\right)}, \frac{\exp \left(a^{L}\right)}{E_{2 n+1}\left(a^{L}-a\right)}\right\}
\end{gathered}
$$

where $n \in \mathbb{N}$ and in the right hand side, only the $n$ 's such that $E_{2 n+1}\left(a^{L}-a\right)>0$ are considered. This defines an ordered Group isomorphism:

$$
\begin{aligned}
\exp :(\mathbf{N o},+,<) & \rightarrow\left(\mathbf{N o}_{>0}, \cdot,<\right) \\
a & \mapsto \exp (a) .
\end{aligned}
$$

The reciprocal isomorphism is denoted:

$$
\begin{aligned}
\log :\left(\mathbf{N o}_{>0}, \cdot,<\right) & \rightarrow(\mathbf{N o},+,<) \\
b & \mapsto \log (b) .
\end{aligned}
$$

which verifies for any $b \in \mathbf{N o}$ :

$$
\begin{aligned}
\log \left(\omega^{b}\right):=\left\{\log \left(\omega^{b^{L}}\right)+n, \log \left(\omega^{b^{R}}\right)-\omega^{\left(b^{R}-b\right) / n} \mid\right. & \\
& \left.\log \left(\omega^{b^{R}}\right)-n, \log \left(\omega^{b^{L}}\right)+\omega^{\left(b-b^{L}\right) / n}\right\} .
\end{aligned}
$$

Moreover, for any $r \in \mathbb{R}, s \in \mathbb{R}_{>0}$ and $a \in \mathbf{N o}, a \prec 1$ :

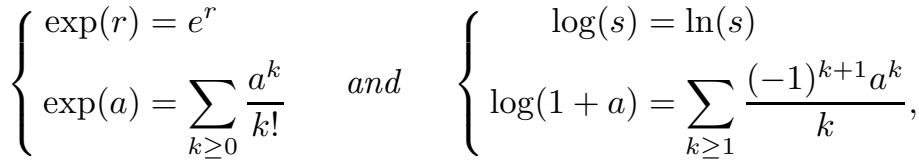

$$
\begin{aligned}
& \left\{\begin{array} { r l } 
{ \operatorname { e x p } ( r ) = e ^ { r } } \\
{ \operatorname { e x p } ( a ) = \sum _ { k \geq 0 } \frac { a ^ { k } } { k ! } }
\end{array} \quad \text { and } \quad \left\{\begin{array}{rl}
\log (s) & =\ln (s) \\
\log (1+a) & =\sum_{k \geq 1} \frac{(-1)^{k+1} a^{k}}{k} .
\end{array}\right.\right.
\end{aligned}
$$


Let us give a hint on how to verify the validity of the inductive formula for exp. Since $E_{n}$ is a truncation of the full series (2.4), one can observe that the elements involved in the cut verify for instance:

$$
\begin{aligned}
\exp \left(a^{L}\right) E_{n}\left(a-a^{L}\right)<\exp \left(a^{L}\right) \cdot \exp \left(a-a^{L}\right) & =\exp (a)= \\
& =\frac{\exp \left(a^{L}\right)}{\exp \left(a^{L}-a\right)}<\frac{\exp \left(a^{L}\right)}{E_{2 n+1}\left(a^{L}-a\right)}
\end{aligned}
$$

whenever only the $n$ 's for which $E_{2 n+1}\left(a^{L}-a\right)>0$ are considered.

The computation of the exponential for finite surreal numbers (i.e. of type $x=$ $r+\epsilon$ for some real $r$ and some infinitesimal $\epsilon$ ) amounts to determining the sum of their power series via (2.4). For purely infinite surreal numbers (i.e. numbers whose Conway normal form has only positive exponents), Gonshor obtains specific results. As an example, let us compute:

$$
\begin{aligned}
\exp (\omega) & =\exp (\{n \mid \emptyset\}) \\
& =\left\{0, \exp (n) \cdot E_{n}(\omega-n) \mid \emptyset\right\} \quad(n \in \mathbb{N}) \\
& \quad\left(\text { since } E_{2 n+1}(n-\omega)<0 \text { for any } n\right) \\
& =\left\{\omega^{n} \mid \emptyset\right\} \quad \text { (by cofinality) } \\
& =\omega^{\omega} .
\end{aligned}
$$

Similarly, one can show that for some of the subsequent infinite ordinals, exp coincides with the omega map, which itself coincides with ordinal exponentiation. However, this is not a general rule. For instance, let us consider the first epsilon number $\varepsilon_{0}=\left\{1, \omega, \omega^{\omega}, \ldots \mid \emptyset\right\}=\left\{\Omega^{n}(0) \mid \emptyset\right\}$. Recall that this is the first fixed point of the ordinal exponentiation: $\omega^{\varepsilon_{0}}=\varepsilon_{0}$. Supposing that we have already proved that exp coincides with $\Omega$ on the infinite ordinals $\Omega^{n}(0), n>1$, we compute as before:

$$
\begin{aligned}
\exp \left(\varepsilon_{0}\right) & =\exp \left(\left\{\Omega^{n}(0) \mid \emptyset\right\}\right) \quad(n \in \mathbb{N}) \\
& =\left\{0, \exp \left(\Omega^{n}(0)\right) \cdot E_{m}\left(\varepsilon_{0}-\Omega^{n}(0)\right) \mid \emptyset\right\} \quad(m \in \mathbb{N}) \\
& =\left\{\Omega^{n+1}(0) \cdot\left(\varepsilon_{0}-\Omega^{n}(0)\right)^{m} \mid \emptyset\right\} \quad \text { (by cofinality) } \\
& =\left\{\omega^{\varepsilon_{0} \cdot m} \mid \emptyset\right\} \quad \text { (since } \omega^{\varepsilon_{0}}=\varepsilon_{0} \text { and again by cofinality) } \\
& =\omega^{\varepsilon_{0} \cdot \omega}=\omega^{\omega^{\varepsilon_{0}+1}} \neq \varepsilon_{0} .
\end{aligned}
$$

The following theorem describes the exact relation between the exponential map and the omega map for purely infinite surreal numbers.

Theorem 2.16 ([Gon86, Theorems 10.8 to 10.13]). Monomials correspond to exponentials of purely infinite surreal numbers:

$$
\omega^{\text {No }}=\exp (\mathbb{J}) \text {. }
$$

Moreover for any purely infinite surreal number $a=\sum_{i<\lambda} \omega^{a_{i}} r_{i}$ (i.e. $\left.\forall i, a_{i}>0\right)$ :

$$
\exp (a)=\omega^{y} \text { with } y=\sum_{i<\lambda} \omega^{g\left(a_{i}\right)} r_{i}
$$

where the map $g: \mathbf{N o}_{>0} \rightarrow \mathbf{N o}$ is defined by:

$$
g(a):=\left\{\operatorname{Ind}(a), g\left(a^{L}\right) \mid g\left(a^{R}\right)\right\} .
$$


The inverse map $\log$ of exp satisfies for any surreal number $b=\sum_{j<\lambda} \omega^{b_{j}} s_{j}$ :

$$
\log \left(\omega^{b}\right)=\log \left(\prod_{j<\lambda}\left(\omega^{\omega^{b_{j}}}\right)^{s_{j}}\right)=\sum_{j<\lambda} \omega^{h\left(b_{j}\right)}
$$

where the map $h: \mathbf{N o} \rightarrow \mathbf{N o}_{>0}$ is defined by:

$$
h(b):=\left\{0, h\left(b^{L}\right) \mid h\left(b^{R}\right), \omega^{b} / 2^{n}\right\} .
$$

We have $h=g^{-1}$ on No.

Subsequently, Gonshor gives detailed results on the map $g$. For instance, he proves in [Gon86, Theorem 10.17] that $g(a)=a$ - equivalently, exp coincides with $\Omega$ on $\omega^{a}$ - for any surreal number $a$ such that $\varepsilon_{\alpha}+\omega \leq a \leq \beta<\varepsilon_{\alpha+1}$ or such that $1 \leq a \leq \beta<\varepsilon_{0}$ for some $\alpha, \beta \in$ On $\left(\varepsilon_{\alpha}\right.$ denotes as usual the $\alpha$ 'th epsilon number). In turn, Gonshor shows that $g$ fails to be the identity map in a close neighborhood of any of the generalized epsilon numbers, i.e. the fixed points for the $\omega$-map [Gon86, Ch.9]. This illustrates a key difference between $\Omega$ and exp, since the latter cannot have fixed points.

As it has been underlined in [vdDE01], the results on No described so far agree with important results on the model theory of the ordered field of real numbers with restricted analytic functions and the exponential function. More precisely, let $L_{\text {an }}(\exp )$ be the language of ordered rings augmented by a symbol for each real multivariate power series convergent on the closed unit hypercube corresponding to their domain, and by a symbol exp, and let $T_{\text {an,exp }}$ be the $L_{\text {an }}(\exp )$-theory of the field of real numbers where the $L_{\text {an }}$ (exp)-symbols are naturally construed as:

- the real analytic functions corresponding to their convergent power series expansion on the hypercube on and extended by 0 outside of it to a welldefined map on $\mathbb{R}$;

- the real exponential map for exp, with the axiomatization proved by Ressayre [Res93].

The corresponding structure $\mathbb{R}_{\text {an,exp }}$ is model complete, o-minimal and has a complete axiomatization with $T_{\text {an, } \exp }$ [Wi196, Second Main Theorem] [vdDMM94, Corollaries 4.5, 4.6 and 5.13]. Recall that these key model theoretic properties have striking geometric counterparts, which are the so-called tameness properties after Grothendieck's [Gro97]: finiteness of the number of connected components, cell decomposition, dimension theory, triangulation,... See [vdD98] and [Cos99]. Using the previously described interpretation of $L_{\mathrm{an}}(\exp )$ and the corresponding results in Gonshor's work, the authors obtain:

Theorem 2.17 ([vdDE01, Theorem 2.1]). The structure $\mathbf{N o}_{\text {an,exp }}$ is an elementary extension of $\mathbb{R}_{\mathrm{an}, \exp }$.

Moreover, the authors describe a family of initial subfields of No, each of which providing an elementary substructure of $\mathbf{N o}_{\text {an, } \exp }$ as well as an elementary extension of $\mathbb{R}_{\mathrm{an}, \exp }$. This means that all these fields share the same first order properties, in particular the geometry based on these non-Archimedean fields is tame.

\section{From Hardy fields to transseries and $H$-FIELdS}

3.1. Hardy fields and tame geometry. Few years after Gonshor's [Gon86], several other similar non standard models of $\mathbb{R}_{\text {an,exp }}$ appeared in two different areas in connection with new important results: 
- in model theory, the field of Logarithmic-Exponential series [vdDMM97, vdDMM94] and the fields of Exponential-Logarithmic series [Kuh00, KS05] related to the results already cited on the theories of $\mathbb{R}_{\exp }$ and $\mathbb{R}_{\mathrm{an}, \exp }$;

- in differential equations, fields of grid-based and well-ordered transseries [vdH97, vdH06, Sch01] related to the proof of the Dulac conjecture [É92].

In any case, one of the main purposes of these objects is to provide formal analogues to non oscillating functions, in the sense of asymptotic scales. In terms of real functions, exp and log have been used at an earlier stage to describe asymptotically non oscillating functions. Many interesting functions have an asymptotic behavior at $+\infty$ that can be described in terms of exp, log and algebraic functions - for instance, the prime number theorem says that $\pi(x) \sim \frac{x}{\log (x)}$, or Stirling's approximation states that $n !=\Gamma(n) \sim \sqrt{2 \pi n} e^{n \log (n)-n}$. Especially, one can obtain asymptotic equivalents at $+\infty$ for solutions of differential equations (Airy functions, hypergeometric functions, etc.).

The theory of such asymptotic expression was eventually formalized by Hardy in his logarithmico-exponential functions (LE-functions) [Har10], after the work of Du Bois-Reymond, with further inspiration from Liouville's work on elementary functions. We paraphrase Hardy's definition using germs instead of actual functions. We define the germ $($ at $+\infty)$ of a real function $f:(a,+\infty) \rightarrow \mathbb{R}$ as the equivalence class:

$$
[f]:=\{g:(b,+\infty) \rightarrow \mathbb{R}: \exists c \in \mathbb{R} \forall x>c(f(x)=g(x))\} .
$$

Germs of real functions form a ring - let us denote it by $\mathcal{G}$ - with the identity given by the constant function $[1]$ and the operations $[f]+[g]=[f+g]$ and $[f \cdot g]=[f] \cdot[g]$.

By definition, the class of logarithmico-exponential germs (LE-germs) is the smallest ring of germs such that:

- the germs of the constant functions and of the identity function $x: \mathbb{R} \rightarrow \mathbb{R}$ are in the ring;

- if $[f]$ is in the class, then $[\exp (f)]$ is in the ring;

- if $[f]$ is in the class and the image of $f$ is strictly positive, then $[\log (f)]$ is in the ring;

- if $[f]$ is in the class, and the image of $f$ is contained in the domain of a semi-algebraic function $A,[A(f)]$ is in the ring.

Note that, by construction, any LE-function $f$ is differentiable in a neighborhood of $+\infty$ and its derivative $f^{\prime}$ is again a LE-function. The mapping $[f] \mapsto\left[f^{\prime}\right]$ is a well-defined derivation on LE-germs. Moreover, as a crucial result [Har10, Theorem, p.18], LE-functions are eventually continuous, of constant sign and monotonic. Subsequently, LE-germs form an ordered field, and eventually a differential ordered field. Therefore, one can compare the order of growth of logarithmico-exponential germs. We define (compare with the definitions given before and after Theorem 2.8):

- $[f] \prec[g]$ if $\lim _{x \rightarrow+\infty} \frac{f}{g}(x)=0$; equivalently, if $n \cdot[f]<[g]$ for all $n \in \mathbb{N}$;

- $[f] \preccurlyeq[g]$ if $[g] \nprec[f]$;

- $[f] \asymp[g]$ if $[f] \preccurlyeq[g]$ and $[g] \preccurlyeq[f]$;

- $[f] \sim[g]$ if $[f]-[g] \prec[f]$ and $[f]-[g] \prec[g]$. 
For instance, we have $\left[e^{x}\right] \succ\left[x^{n}\right]$ for all $n \in \mathbb{N}$, while $\left[e^{x}\right] \sim\left[e^{x}\right]+[\log (x)]$, and so on. In fact, for any two germs $[f],[g]$, one has either $[f] \prec[g],[g] \prec[f]$, or $[f] \sim[r g]$ for some non-zero real number $r \in \mathbb{R}$.

LE-germs represent a large asymptotic scale, which includes the usual scale consisting of powers of $x$, and that has still the rich algebraic structure of an ordered differential field. Building on Hardy's work, Bourbaki introduced the following general notion:

Definition 3.1 ([Bou76, p.V-36]). A Hardy field is a subring of $\mathcal{G}$ that is a field and that is closed under differentiation.

For instance, the field of (germs of) rational fractions with real coefficients $\mathbb{R}(x)$ is a Hardy field. So is the field of functions real meromorphic at infinity $\mathbb{R}\{1 / x\}$. There are various ways to derive new Hardy fields from a given one $K$ : by taking its real closure $K^{r c}$ in $\mathcal{G}$ [Rob72]; by taking its Liouville closure (i.e. closing under integration and logarithmic integration) $K^{l c}$ in $\mathcal{G}$ [Bou76]; by taking its closure under resolution of differential equations $Q(y) y^{\prime}=P(y)$ for $P, Q \in K[y]$ [Ros83, based on an unpublished work by M. Singer]; by adjoining solutions of certain second order linear differential equations [Bos87, Ros95]. Note that the field of LE-germs is not closed under the latter extensions; by Liouville's theorem, it does not contain the antiderivative of $e^{-x^{2}}$.

In fact, Hardy fields tends to be central objects in tame geometry: given an o-minimal structure over $\mathbb{R}$, the germs at $+\infty$ of unary real functions that are definable (i.e. whose graph is a subset definable in the structure) form a Hardy field. Indeed, differentiation is a definable operation, and functions are ultimately non oscillating by o-minimality. The simplest examples are the Hardy fields corresponding to the structure of semialgebraic sets and to subanalytic sets: they consist in Puiseux power series at $+\infty$ with real coefficients and that are algebraic, respectively convergent. Another key example, which contains both the preceding examples and the field of LE-germs, is the Hardy field corresponding to $\mathbb{R}_{\mathrm{an} \text {,exp }}$. Note that, in this case also, one has an explicit description of these germs in terms of compositions of exp, log and restricted real analytic functions [vdDMM94, Corollary 4.7].

Nevertheless, understanding Hardy fields in full generality remains a challenging problem. Generally speaking, the union of two Hardy fields does not generate a Hardy field. It implies that there is no maximum Hardy field, but several maximal ones. Furthermore, there exist Hardy fields that contain functions growing more than any iteration of the exponential function [Bos86]. Several questions remain unanswered: how do maximal Hardy fields look like? What differential equations can be solved in them? Are there maximal Hardy fields corresponding to some o-minimal structure?

3.2. From the standpoint of formal power series. As we already announced, there are several similar non standard models of the theory of $\mathbb{R}_{\text {an,exp }}$ based on generalized power series: fields of LE-series, EL-series and transseries. All of them are special kinds of ordered fields of formal power series, which carry exponential and logarithmic maps. Recall that, according to [KKS97], there is no hope for defining a global exponential function directly on a full field of generalized power series $\mathbb{R}((\mathfrak{M})$ ) (where $\mathfrak{M}$ denotes the (multiplicatively written) ordered Abelian group of (generalized) monomials). Indeed, such exp should give an isomorphism 
between the additive group and the positive part of the multiplicative group. By decomposing the additive group as in (2.2) and the multiplicative group as in (2.3), one can verify that the isomorphism induces three isomorphisms between the three components of the decompositions. However, even though such isomorphisms exist in any generalized series field on the elements of non negative valuation (i.e., the "finite series", compare with Theorem 2.15), there cannot be any between the additive group of series having terms with negative value (i.e. "purely infinite" series) and the multiplicative group of monomials $\mathfrak{M}$, as proven in [KKS97]. Thus, no global exponential function exist.

Hence, one has to use a special construction to obtain such isomorphism in the context of generalized power series. The main common idea in the different versions is due independently to [D84, DG87] and [É92], and may be seen as an abstract version of Hardy's construction of log-exp functions [Har10]. It consists in building extensions of a given $\mathbb{R}((\mathfrak{M}))$ which will contain the missing elements to obtain a well-defined exp and a surjective log: the corresponding field with global exp and $\log$ is then obtained as a subfield of such an enlarged field of generalized series. Let us describe the process for the exponential closure of $\mathbb{R}((\mathfrak{M}))$ :

- Any field of generalized series $\mathbb{R}((\mathfrak{M}))$ has a partial exponential map $e$ defined on the series with non negative value (the "finite part" of the series) using the real exponential map and the Taylor formula (2.4).

- Given a field of generalized series $(\mathbb{R}((\mathfrak{M})), e)$ endowed with a partial exponential map $e$, we consider $\mathfrak{M}^{\sharp}=: e^{\sharp}\left(\mathbb{R}\left(\left(\mathfrak{M}_{\succ 1}\right)\right)\right)$ a multiplicative copy of the group of purely infinite series where the morphism $e^{\sharp}$ is intended to extend $e$. More precisely, the image of $e$ and its complement are ordered lexicographically. Accordingly, the exponential extension of $(\mathbb{R}((\mathfrak{M})), e)$ is the field $\left(\mathbb{R}\left(\left(\mathfrak{M}^{\sharp}\right)\right), e^{\sharp}\right)$ for $e^{\sharp}$ the corresponding function extension of $e$.

- Considering infinitely many iterated exponential extensions of the given series field $\mathbb{R}((\mathfrak{M}))$, its exponential closure - denote it by $\mathbb{R}((\mathfrak{M}))^{\mathrm{E}}$ - is the direct limit of such tower of fields with partial exponentials. The morphism obtained as a limit of the partial $e$ 's is a surjective exponential that we denote by exp.

Note that $\mathbb{R}((\mathfrak{M}))^{\mathrm{E}}$ is a subfield of the generalized series field $\mathbb{R}\left(\left(\mathfrak{M}^{\mathrm{E}}\right)\right)$ where $\mathfrak{M}^{\mathrm{E}}$ denotes the union of the iterated extended groups of monomials. Concerning the logarithmic closure, different approaches provide different objects: see [KT12] for a comparison between LE-series and EL-series. There are also variants in case one imposes restrictions on the supports of the powers series considered: compare gridbased and well-ordered transseries in [vdH97]. Note also that restricted analytic functions extend to such various fields as they extend to generalized power series fields (direct consequence of Neumann's Lemma [N49]).

A key additional feature of these non standard models of $\mathbb{R}_{\mathrm{an}, \exp }$ is that they can be endowed with particular nice derivations (provided that the initial field $\mathbb{R}((\mathfrak{M})$ ) carries such a derivation):

- that agree with the structure of power series, in particular which commute with infinite sums [vdDMM01, Section 3] [Sch01, Definition 4.1.1] [KM11, Definition 3.6];

- that are compatible with the exp and log functions, namely $D(\exp (x))=$ $\exp (x) \cdot D(x)$ and $x \cdot D(\log (x))=D(x)$; 
- that behave very like the derivation of a Hardy field, in particular like the Hardy field associated to $\mathbb{R}_{\mathrm{an}, \exp }$ (see [vdDMM01, Section 4] [KM11, Definition 4.1] and the following section).

Recall that these power series with exp and log are meant to be formal counterparts to actual non oscillating functions, in particular in the context of differential equations. This will be discussed further later on. Let us just state already the following striking embedding theorem which follows directly from the cited model theoretic results on $\mathbb{R}_{\mathrm{an}, \exp }$ :

Theorem 3.2 ([vdDMM01, Corollary 3.12]). The Hardy field associated to $\mathbb{R}_{\mathrm{an}, \exp }$ embeds naturally as a differential exponential and logarithmic real closed field into the field of LE-series.

In [Sch01], the author proposes an axiomatic version of transseries. In order to avoid confusion, we slightly modify the terminology used by this author:

Definition 3.3. Let $\mathbb{R}((\mathfrak{M}))$ be a field of generalized series and log be a function such that:

(T1): the domain of log consists of the positive series;

(T2): $\log (\mathfrak{M}) \subseteq \mathbb{R}\left(\left(\mathfrak{M}_{\succ 1}\right)\right)$;

(T3): $\log (1+\varepsilon)=\sum_{n \geq 1}(-1)^{n+1} \frac{\varepsilon^{n}}{n}$ for any $\varepsilon \in \mathbb{R}\left(\left(\mathfrak{M}_{\preccurlyeq 1}\right)\right)$;

(T4): let $\left(\mathfrak{m}_{n}\right)_{n \in \mathbb{N}}$ be a sequence of monomials with $\mathfrak{m}_{n+1}$ in the support of $\log \left(\mathfrak{m}_{n}\right)$ for all $n$. There is an integer $n_{0}$ such that for any $n \geq n_{0}$, for any $\mathfrak{m}$ in the support of $\log \left(\mathfrak{m}_{n}\right)$, one has that $\mathfrak{m} \succcurlyeq \mathfrak{m}_{n+1}$ and the coefficient of $\mathfrak{m}_{n+1}$ in $\log \left(\mathfrak{m}_{n}\right)$ is \pm 1 .

Note that such axioms remain verified by the corresponding transseries field $\mathbb{R}((\mathfrak{M}))^{\mathrm{E}}$. In fact, the author does even consider transfinite exponential extensions. Axioms (T1) to (T3) mean that $\mathbb{R}((\mathfrak{M}))$ is endowed with a so-called prelogarithm [Kuh00]. (T4) takes into account the possible existence of elements of type:

$$
y=\exp \left(x+\exp \left(\log _{2}(x)+\exp \left(\log _{4}(x)+\ldots\right)\right)\right),
$$

the latter being viewed as a formal solution to the functional equation: $y=\exp (x+$ $\left.\log _{2}(y)\right)(x$ is construed as the germ of identity at $+\infty)$. Nevertheless, (T4) forbids the existence of series of type:

$$
\exp \left(x+\exp \left(\log _{2}(x)+\exp \left(\log _{4}(x)+\cdots+\log _{6}(x)\right)+\log _{4}(x)\right)+\log _{2}(x)\right) .
$$

The reader should compare this with the notion of irreducible surreal numbers in [Con76, p. 33]. In [KM15], the authors introduced the more restrictive notion of field of exp-log transseries as a common axiomatic for EL-series and transseries, where (T4) is replaced by:

(ELT4): let $\left(\mathfrak{m}_{n}\right)_{n \in \mathbb{N}}$ be a sequence of monomials with $\mathfrak{m}_{n+1}$ in the support of $\log \left(\mathfrak{m}_{n}\right)$ for all $n$. There is an integer $n_{0}$ such that $\log \left(\mathfrak{m}_{n_{0}+k}\right)=\mathfrak{m}_{n_{0}+k+1}$ for any $k \in \mathbb{N}$.

As will be discussed with further details in Section 4 this axiom applies to an important strict subfield of No, but not to No itself. 
3.3. $H$-fields as a common axiomatic framework. Concerning Hardy fields as described in Section 3.1, Rosenlicht studied the remarkable properties linking the derivation and the ordering as a differential ordered fields - or accordingly the corresponding natural valuation $v$ as a differential valued fields [Ros83]. Building on his work, M. Aschenbrenner and L. van den Dries introduced in [AD02] the following more abstract notion:

Definition 3.4. An $H$-field is an ordered field $(K,+, \cdot, \leq)$ equipped with a function $D: K \rightarrow K$ such that:

(1) additivity: $D(x+y)=D(x)+D(y)$;

(2) Leibniz rule: $D(x y)=x D(y)+y D(x)$;

(3) H1: if $x>\operatorname{ker}(D)$, then $D(x)>0$;

(4) H2: if $|x|<c$ for some $c \in \operatorname{ker}(D)$, then there exists $d \in \operatorname{ker}(D)$ such that $|x-d|<c$ for all $c \in \operatorname{ker}(D)$.

For a Hardy field $K$, Condition H1 follows from the fact that if $[f]>\mathbb{R}$, then $\lim _{x \rightarrow+\infty} f(x)=+\infty$, which implies that $f^{\prime}(x)$ is eventually positive, so $\left[f^{\prime}\right]>0$. Condition H2 holds whenever $\operatorname{ker}\left(\frac{d}{d x}\right)=\mathbb{R} \subseteq K$.

Given an $H$-field $K$ with derivation $D$, the convex hull of $\operatorname{ker}(D)$ is a valuation ring. Let us denote by $v: K^{\times} \rightarrow \Gamma$ the corresponding valuation, where $\Gamma$ is a suitable ordered Abelian group $(v(x) \geq 0$ if and only if $x$ is in the convex hull of $\operatorname{ker}(D))$. We shall use again the notation of Hardy and Du Bois-Reymond accordingly to this valuation (compare with Section 3.1). For $x, y \in K^{\times}$, we shall write:

- $x \prec y$ if $v(x)>v(y)$;

- $x \preceq y$ if $v(x) \geq v(y)$;

- $x \asymp y$ if $v(x)=v(y)$;

- $x \sim y$ if $v(x-y)>v(x)$ and $v(x-y)>v(y)$.

Derivation and valuation are linked via specific properties. For the sake of notation, we shall denote by $L D(x)$ the logarithmic derivative $L D(x):=D(x) / x$. In particular, one has:

- a strong version of L'Hospital rule: $\forall x, y \nprec 1,(x \prec y \Leftrightarrow D(x) \prec D(y))$;

- a rule for $L D: \forall x \prec y \prec 1, L D(x) \succcurlyeq L D(y)$.

Note that these properties were also used in [KM11, KM12] by the authors as axioms of a so-called Hardy type derivation for a differential valued field.

In most cases, one also requires that the derivation preserves infinitesimal elements.

Definition 3.5. An $H$-field $(K, D)$ has small derivation if $D(x) \prec 1$ for all $x \in K$ such that $x \prec 1$.

Note that this assumption is rather harmless. Indeed, for elements $y, z \in K, z \succ$ 1 , such that $y=D(z)$, if we set $D^{y}: x \mapsto y \cdot D(x)$, then $\left(K, D^{y}\right)$ is an $H$-field with small derivation. Note also that all Hardy fields with their natural derivation $\frac{\mathrm{d}}{\mathrm{d} x}$ are $H$-fields with small derivation ([f] infinitesimal means that $\lim _{x \rightarrow+\infty} f(x)=0$, which implies that $\lim _{x \rightarrow+\infty} f^{\prime}(x)=0$, so $\left[f^{\prime}\right]$ is infinitesimal as well).

Rosenlicht observed in [Ros81] that some of the key relations between the derivation $D$ and the valuation $v$ can be encoded within the valued group $\Gamma$ equipped with an extra function that we denote also by $L D$ : for $\gamma=v(a) \in \Gamma^{\neq 0}$, let 
$L D(\gamma):=v(L D(a))=v(D(a) / a)=v(D(a))-\gamma$. Such $(\Gamma, L D)$ is called an asymptotic couple in the context of Hardy fields. In [AD02], the authors enhance this study of asymptotic couples for $H$-fields. In particular, of prime interest is the following notion related to the question of integration:

Definition 3.6. Let $(K, D)$ be an $H$-field. Given some $a \in K$, an element $b \in K$ is an asymptotic integral of $a$ if $a \sim D(b)$.

Theorem 3.7 ([Ros83]). Let $(K, D)$ be an $H$-field, with asymptotic couple $(\Gamma, L D)$. For all $a \in K, v(a)$ is not the supremum of $L D\left(\Gamma^{\neq 0}\right)$ if and only if a admits an asymptotic integral in $K$.

Corollary 3.8. Let $(K, D)$ be an $H$-field, with asymptotic couple $(\Gamma, L D)$. If $L D\left(\Gamma^{\neq 0}\right)$ has no supremum in $\Gamma$, then every element of $K$ admits an asymptotic integral.

When all elements of $K$ admits an asymptotic integral, we say that $K$ admits asymptotic integration. This notion applies particularly in the context of spherically complete fields, the abstract version of fields of generalized power series (see Section 2.2).

Theorem 3.9 ([Kuh11]). Let $K$ be an $H$-field. If every $a \in K$ admits an asymptotic integral, and $K$ is spherically complete, then every $a \in K$ admits an integral.

Indeed, if one wants to find an integral of a given element $a \in K$, one can define a sequence of approximations as follows: first, we let $b_{0}$ be an asymptotic integral of $a$, then we define inductively $b_{i+1}$ to be the asymptotic integral of $D\left(b_{i}\right)-a$. Provided one takes appropriate precautions, such as using $\mathrm{H} 2$ to ensure that $v\left(b_{i}\right) \neq 0$ for all $i$, the resulting sequence $\left(b_{i}\right)$ is pseudo-Cauchy, and when $K$ is spherically complete, it has a pseudo-limit $b_{\omega}$. If $D\left(b_{\omega}\right)=a$, we are done. If not, we keep iterating on all ordinals. The procedure eventually produces an integral for $a$.

As mentioned before, Hardy fields can be closed under algebraic extensions and application of exp and $\log$ via the notion of Liouville closure (see Section 3.1). Similarly, every $H$-field can be extended to a Liouville-closed $H$-field, that is to say, a real closed $H$-field in which the equations $y^{\prime}=a$ and $z^{\prime}=a z \wedge z \neq 0$ have solutions for all $a$. Moreover, suppose now that the $H$-field $K$ is also equipped with an exponential function exp : $K \rightarrow K^{>0}$ compatible with the derivation $D$, namely such that $D(\exp (x))=\exp (x) D(x)$ for all $x \in K^{>0}$. Then $K$ is Liouville-closed if and only if the equation $y^{\prime}=a$ has a solution for all $a \in K$. Indeed, to solve $z^{\prime}=a z \wedge z \neq 0$ it suffices to take a $b \in K$ such that $D(b)=a$ and note that $\exp (b)$ is a solution to $z^{\prime}=a z$.

Theorem 3.10 ([AD02]). Every $H$-field has one or two Liouville closures up to isomorphism.

In many important cases, the number of Liouville closures of an $H$-field $K$ is determined by the structure of its asymptotic couple (however, A. Gehret proved recently that when $K$ has asymptotic integration, the determining factor for the number of Liouville closures is not the asymptotic couple, but rather the property of " $\lambda$-freeness" of $K$ [G16]). In [ADH1], the authors identified a key first order axiom that applies to the field of LE-series and which implies uniqueness of the Liouville closure. 
Definition 3.11. An $H$-field $(K, D)$ is $\omega$-free if it is real closed, it admits asymptotic integration, and

$$
\forall a \exists b\left(b \succ 1 \wedge a-\omega(L D(L D(b))) \succeq L D(b)^{2} .\right.
$$

where $\omega(y):=-\left(2 y^{\prime}-y^{2}\right)$.

$\omega$-freeness relates to the solvability within $K$ of second order linear equations of type: $4 y^{\prime \prime}+f y=0$ which is governed by the behaviour of the corresponding Riccati operator $\omega$. This is already discussed in [Ros95] in the context of Hardy fields.

Theorem 3.12 ([ADH1, Corollary 13.6.2]). If an $H$-field $K$ is $\omega$-free, then it has a unique Liouville closure up to isomorphism.

We conclude this section by quoting one of the main theorems of Aschenbrenner, van den Dries and van der Hoeven in [ADH1], after having been conjectured by the same authors 20 years ago. The other key notion is the one of Newtonianity, a version with asymptotic constraints of differential Henselianity: any asymptotic algebraic differential equation of Newton degree one has a root in the valuation ring.

Theorem 3.13 ([ADH1]). Let $T$ be the first-order theory in the language $\mathcal{L}=$ $\{0,1,+, \cdot,<, \prec, D\}$ whose models are the $H$-fields $K$ such that:

- the derivation is small;

- $K$ is Liouville-closed;

- $K$ is $\omega$-free;

- $K$ is Newtonian.

Then $T$ is complete and model-complete. Moreover, the fields of LE-series and grid-based transseries are a model of $T$.

We can now state the relevant related questions: can No be given the structure of an $H$-field? Is it a model of the theory $T$ ? Is it universal?

\section{Surreal Derivations}

Inspired by the definition of $H$-field, and by the properties of derivations on fields of transseries, the authors define in [BM] derivations on No as follows.

Definition 4.1. A surreal derivation is a function $D:$ No $\rightarrow$ No satisfying the following properties:

(1) Leibniz rule: $D(x y)=x D(y)+y D(x)$;

(2) strong additivity: $D\left(\sum_{i \in I} x_{i}\right)=\sum_{i \in I} D\left(x_{i}\right)$ if $\left(x_{i}: i \in I\right)$ is summable;

(3) compatibility with exponentiation: $D(\exp (x))=\exp (x) D(x)$;

(4) constant field $\mathbb{R}: \operatorname{ker}(D)=\mathbb{R}$;

(5) $H$-field: if $x>\mathbb{R}$, then $D(x)>0$.

Recall that surreal numbers can be written in normal form as

$$
x=\sum_{i<\lambda} \omega^{x_{i}} \cdot r_{i}=\sum_{i<\lambda} e^{\gamma_{i}} \cdot r_{i}
$$

with $\gamma_{i} \in \mathbb{J}$. 
A naive attempt to construct a derivation $D$ is to proceed by induction, using the following identity, which is a consequence of rules (2), (3) and (4):

$$
D(x)=D\left(\sum_{i<\lambda} e^{\gamma_{i}} \cdot r_{i}\right)=\sum_{i<\lambda} e^{\gamma_{i}} \cdot r_{i} \cdot D\left(\gamma_{i}\right) .
$$

There are two obstructions, however, to such approach: the existence of logatomic numbers, and finding a way of working by induction.

\subsection{Log-atomic numbers.}

Definition 4.2. A surreal number $x \in \mathbf{N o}$ is log-atomic if for all $n \in \mathbb{N}$ there exists $y_{n} \in$ No such that

$$
\underbrace{\log (\ldots(\log }_{n \text { times }}(x)) \ldots)=\omega^{y_{n}} .
$$

We let $\mathbb{L}$ be the class of all log-atomic numbers.

For instance, the ordinal numbers $\omega$ and $\epsilon_{0}$ are log-atomic numbers; in fact, all $\epsilon$-numbers are log-atomic [Gon86]. If $x$ is log-atomic, then (4.1) does not provide information on its own regarding the value of $D(x)$; for instance, it does not tell anything about the value we should give to $D\left(\epsilon_{0}\right)$. It is useful at this point to classify log-atomic numbers. The first step in this direction was done in [KM15] using a strategy inspired by Conway's definition of the $\Omega$-map.

Definition 4.3 ([KM15]). Given two positive infinite surreal numbers $x, y \in \mathbf{N o}^{>\mathbb{N}}$, we say that $x$ and $y$ have the same exp-log class, and we write $x \sim^{K} y$, if there exists some $n \in \mathbb{N}$ such that

$$
\underbrace{\log (\ldots(\log }_{n \text { times }}(x)) \ldots)<y<\underbrace{\exp (\ldots(\exp }_{n \text { times }}(x)) \ldots) .
$$

A $\kappa$-number is a surreal number that is the simplest in its own exp-log class. Just as monomials can be parametrised by the $\Omega$-map, $\kappa$-numbers can be similarly parametrised by the $\kappa$-map. For the sake of notation, for $n \in \mathbb{N}$, we define inductively $\log _{0}(x)=x, \log _{n+1}(x)=\log \left(\log _{n}(x)\right)$, and similarly $\exp _{0}(x)=x$, $\exp _{n+1}(x)=\exp \left(\exp _{n}(x)\right)$. Define

$$
\kappa_{x}:=\left\{n, \exp _{n}\left(x^{\prime}\right) \mid \log _{n}\left(x^{\prime \prime}\right)\right\}
$$

as $n$ varies in $\mathbb{N}$.

We have $\kappa_{0}=\omega, \kappa_{1}=\epsilon_{0}$. Each $\kappa$-number is of the form $\kappa_{x}$ for some $x \in$ No. In [KM15] the authors gave a detailed description of the sign sequence of $\kappa_{x}$ in term of the sign sequence of $x$, which in turn can be used to show that all $\kappa$-numbers are log-atomic.

Theorem 4.4 ([KM15]). All $\kappa$-numbers, and their images via $\exp _{n}, \log _{n}$, are logatomic.

However, the converse does not hold. To capture all log-atomic numbers, one needs to use the finer notion of level adapted from Hardy fields [Ros87, MM97].

Definition 4.5. Given two positive infinite surreal numbers $x, y \in \mathbf{N o}^{>\mathbb{N}}$, we say that $x$ and $y$ have the same level, and we write $x \sim^{L} y$, if there exists some $n \in \mathbb{N}$ such that

$$
\log _{n}(x) \sim \log _{n}(y)
$$


As for exp-log classes, a $\lambda$-number is a surreal number that is the simplest in its own level. Again, $\lambda$-numbers can be parametrised via the $\lambda$-map:

$$
\lambda_{x}:=\left\{n, \exp _{n}\left(n \cdot \log _{n}\left(x^{\prime}\right)\right) \mid \exp _{n}\left(\frac{1}{n} \cdot \log _{n}\left(x^{\prime \prime}\right)\right)\right\}
$$

as $n$ varies in $\mathbb{N}^{*}$.

We have $\lambda_{0}=\omega, \lambda_{1}=\exp (\omega), \lambda_{\omega}=\epsilon_{0}$. Each $\lambda$-number is of the form $\lambda_{x}$ for some $x \in$ No.

Theorem 4.6 ([BM]). All $\lambda$-numbers are log-atomic, and all log-atomic numbers are $\lambda$-numbers.

Moreover, the identity $\lambda_{x+1}=\exp \left(\lambda_{x}\right)$ holds for all $x \in$ No [ADH2], and comparing this equation with the definition of $\kappa$-number, one can verify that $\lambda_{x}$ is a $\kappa$-number if and only if $x \in \mathbb{J}$.

Note that a priori, surreal derivations can take almost arbitrary values on $\mathbb{L}$, subject to very few restrictions: for all $\lambda, \mu \in \mathbb{L}$, one must have $D(\exp (\lambda))=$ $\exp (\lambda) D(\lambda)$, and if $\lambda<\mu$, then $0<D(\lambda)<D(\mu)$. A further restriction imposed by the definition of surreal derivation is that one must have $\log (D(\lambda))-\log (D(\mu)) \prec$ $\max \{\lambda, \mu\}$ for all distinct $\lambda, \mu \in \mathbb{L}$.

As in [BM], we now define $\partial_{\mathbb{L}}: \mathbb{L} \rightarrow$ No as the simplest function respecting the above restrictions. It turns out that such function $\partial_{\mathbb{L}}$ can be calculated quite explicitly. Given a surreal number $x \in \mathbf{N o}$, let $\alpha \in \mathbf{O n}$ be the least purely infinite ordinal number such that $\lambda_{-\alpha} \geq-x$; then

$$
\begin{aligned}
\partial_{\mathbb{L}}\left(\lambda_{x}\right)=\exp \left(\sum_{i=1}^{\infty} \log _{i}\left(\lambda_{x}\right)-\sum_{\beta<\alpha+1} \lambda_{-\beta}\right)= \\
=\exp \left(\sum_{i=1}^{\infty} \log _{i}\left(\lambda_{x}\right)-\sum_{\beta<\gamma+1} \sum_{i=1}^{\infty} \log _{i}\left(\kappa_{-\beta}\right)\right)
\end{aligned}
$$

where $\gamma$ is the unique ordinal such that $\lambda_{-\alpha}=\kappa_{-\gamma}$. Note for instance that we obtain $\partial_{\mathbb{L}}(\omega)=\partial_{\mathbb{L}}\left(\lambda_{0}\right)=1$.

4.2. Extending $\partial_{\mathbb{L}}$ to No. Let $\mathbb{R}\langle\langle\mathbb{L}\rangle\rangle$ be the smallest subfield of No containing $\mathbb{R}, \mathbb{L}$, closed under exp, log, and infinite sums. It is immediate from the definition that if $\partial_{\mathbb{L}}$ extends to a surreal derivation, then its values on $\mathbb{R}\langle\langle\mathbb{L}\rangle\rangle$ are already determined by the values of $\partial_{\mathbb{L}}$ on $\mathbb{L}$. Since $\mathbb{R}\langle\langle\mathbb{L}\rangle\rangle$ can be constructed inductively from $\mathbb{R}$ and $\mathbb{L}$ by taking successive closures, (4.1) does provide an inductive definition of such values. One still has to check that the definition is well posed, namely that the infinite sums appearing on the right hand side are indeed summable. This can be done, for instance, by verifying that $\partial_{\mathbb{L}}$ can be extended first to $\mathbb{R}((\mathbb{L}))$, and then by applying the results of [Sch01].

The fact that (4.1) provides an inductive definition of the extension of $\partial_{\mathbb{L}}$ to $\mathbb{R}\langle\langle\mathbb{L}\rangle\rangle$ is due to the fact that $\mathbb{R}\langle\langle\mathbb{L}\rangle\rangle$ satisfies the condition (ELT4) as mentioned in Section 3.2; in fact, $\mathbb{R}\langle\langle\mathbb{L}\rangle\rangle$ is the largest subfield of No that is also a field of EL-series in the sense of [KM15] (see [BM]).

However, No is strictly larger than $\mathbb{R}\langle\langle\mathbb{L}\rangle\rangle$, as it contains numbers as

$$
x=\exp \left(\omega+\exp \left(\log _{2}(\omega)+\exp \left(\log _{4}(\omega)+\ldots\right)\right)\right)
$$


which are solutions to the fixed point equation $f=\exp \left(\omega+\log _{2}(f)\right)$ and which do not verify (ELT4).

On the other hand, No does satisfy the weaker combinatorial principle (T4) isolated in [Sch01] and is therefore a field of transseries (see Definition 3.2). Applying essentially the same technique that Schmeling used to treat exponential extensions, one can prove that Condition (T4) guarantees that the right hand side of (4.1) remains summable $[\mathrm{BM}]$, so $\partial_{\mathbb{L}}$ does extend to a surreal derivation. While the extension of $\partial_{\mathbb{L}}$ from $\mathbb{L}$ to $\mathbb{R}\langle\langle\mathbb{L}\rangle\rangle$ is unique, its further extension to No may not be. Still, there is a "simplest" extension $\partial:$ No $\rightarrow$ No.

Theorem 4.7 ([BM]). There exist several surreal derivations, among which a "simplest" one $\partial:$ No $\rightarrow$ No extending $\partial_{\mathbb{L}}$.

4.3. Universality of $(\mathbf{N o}, \partial)$. It turns out that the derivation $\partial$ has very good properties. Since $\partial$ is a surreal derivation with $\partial(\omega)=1,(\mathbf{N o}, \partial)$ is an $H$-field with small derivation.

We now note that the asymptotic couple of the field $(\mathbf{N o}, \partial)$ is actually $(\mathbb{J}, \partial)$, up to reversing the ordering of $\mathbb{J}$, and that $L D\left(\mathbb{J}^{\neq 0}\right.$ ) has no infimum in $\mathbb{J}$ (recall that $\mathbb{J}$ is the non-unital ring of purely infinite numbers). For the latter, it suffices to check that $L D(\mathbb{L})=\partial(\mathbb{L})=\partial_{\mathbb{L}}(\mathbb{L})$ has no infimum in $\mathbb{J}$. By the aforementioned Theorem 3.7 by Rosenlicht, we obtain the following:

Proposition 4.8. The differential field $(\mathbf{N o}, \partial)$ admits asymptotic integration.

On the other hand, since No can be presented as a union of spherically complete fields, each one closed under asymptotic integration, one can deduce that $(\mathbf{N o}, \partial)$ is closed under integration using F.-V. Kuhlmann's Theorem 3.9. Since No also has an exponential function that by construction of $\partial$ is compatible with the derivation, this implies the following:

Theorem 4.9 ([BM]). The differential field $(\mathrm{No}, \partial)$ is Liouville-closed.

A similar, although way more subtle argument by Aschenbrenner, van den Dries and van der Hoeven has been used to show that $(\mathbf{N o}, \partial)$ is in fact a model of the theory of LE-series, and in turn that it is universal among all $\mathrm{H}$-fields with constant field $\mathbb{R}$ and small derivation [ADH2].

Theorem 4.10 ([ADH2]). The field $(\mathbf{N o}, \partial)$ is an elementary extension of the $H$-field of LE-series (when identifying the LE-series $x$ with the number $\omega$ ).

The strategy to prove that $(\mathbf{N o}, \partial)$ is an elementary extension of LE-series is in fact the same the authors of [ADH1] use to prove that the field of LE-series is a model of the theory of Theorem 3.13. Say that an $H$-field $(K, D)$, with asymptotic couple $(\Gamma, L D)$, is grounded if $L D\left(\Gamma^{\neq 0}\right)$ has a maximum. If one can present an $H$ field closed under integration as a directed union of spherically complete, grounded sub- $H$-field, then the $H$-field is in fact both $\omega$-free and Newtonian [ADH1, Cor. 11.7.15, Thm. 15.0.1]. The first result follows from the fact that groundedness implies rather directly $\omega$-freeness, which in turn is preserved by directed unions. Newtonianity follows by a more delicate argument: if the derivation is surjective in a tower of spherically complete grounded $H$-fields, then for any asymptotic differential equation defined at some stage of the tower, a solution in the valuation ring appears few steps later in the tower (in fact, under some mild assumptions, the number of steps needed is the order of the differential equation). 
To conclude that $(\mathbf{N o}, \partial)$ is a model of the theory of Theorem 3.13, one writes No as the union of $K_{\epsilon}$, for $\epsilon$ running over the ordinal epsilon numbers (i.e. such that $\left.\omega^{\epsilon}=\epsilon\right)$, where $K_{\epsilon}$ is the set of the surreal numbers of the form

$$
\sum_{i<\lambda} r_{i} \prod_{j<\mu}\left(\omega^{\omega^{a_{i j}}}\right)^{s_{i j}}
$$

such that either the sign sequence of each $a_{i j}$ is of length less than $\epsilon$, or $a_{i j}=-\epsilon$.

Each $K_{\epsilon}$ is a spherically complete field, and one can verify that it is closed under $\partial$, and it is grounded by construction. This shows that $(\mathbf{N o}, \partial)$ is a model of the theory of LE-series; by model-completeness of such theory, it is in fact an elementary extension. Moreover, thanks to the explicit quantifier elimination described in [ADH1], one can in fact prove that $(\mathbf{N o}, \partial)$ is a universal $H$-field:

Theorem 4.11 ([ADH2]). Every $H$-field with small derivation and constant field $\mathbb{R}$ - in particular every Hardy field containing $\mathbb{R}$ - can be embedded over $\mathbb{R}$ as an ordered differential field into No.

\section{REFERENCES}

[All87] N. L. Alling, Foundations of analysis over surreal number fields. North-Holland Mathematics Studies 141, North-Holland Publishing Co., Amsterdam, 1987.

[AD02] M. Aschenbrenner, L. van den Dries, $H$-fields and their Liouville extensions, Mathematische Zeitschrift 3 (2002), 543-588.

[ADH1] M. Aschenbrenner, L. van den Dries and J. van der Hoeven, Asymptotic Differential Algebra and Model Theory of Transseries. arXiv:1509.02588v2 [math.LO].

[ADH2] M. Aschenbrenner, L. van den Dries and J. van der Hoeven, The surreal numbers as a universal H-field. arxiv:1512.02267 [math.LO].

[BM] A. Berarducci and V. Mantova, Surreal numbers, derivations and trasseries, J. Eur. Math. Soc. To appear.

[Bos86] M. Boshernitzan, Hardy fields and existence of transexponential functions. Aequationes Math. 30 (1986) no. 2-3, 258-280.

[Bos87] M. Boshernitzan, Second order differential equations over Hardy fields, J. London Math. Soc. (2) 35 (1987), no. 1, 109-120.

[Bou76] N. Bourbaki, Éléments de mathématique. Fonctions d'une variable réelle, Théorie élémentaire. Nouvelle éd. Hermann, Paris, 1976.

[Con76] J. H. Conway, On numbers and games, second ed., A. K. Peters Ltd., Natick, MA, 2001.

[Cos99] M. Coste, An introduction to o-minimal geometry, Dipartimento di Matematica Dell'Universita di Pisa, 1999.

[CEF] O. Costin, P. Ehrlich and H.M. Friedman, Integration on the surreals: a conjecture of Conway, Kruskal and Norton. arXiv:1505.02478 [math.LO].

[D84] B. I. Dahn, The limit behaviour of exponential terms. Fund. Math. 124 (1984) no. 2, 169-186.

[DG87] B. I. Dahn and P. Göring, Notes on exponential-logarithmic terms. Fund. Math. 127 (1987) no. 1, 45-50.

[vdD98] L. van den Dries, Tame topology and o-minimal structures. Cambridge University Press, Cambridge, 1998

[vdDE01] L. van den Dries and P. Ehrlich, Fields of surreal numbers and exponentiation, Fund. Math. 167 (2001), no. 2, 173-188.

[vdDMM94] L. van den Dries, A. Macintyre, and D. Marker, The elementary theory of restricted analytic fields with exponentiation. Ann. of Math. (2) 140 (1994) no. 1, 183-205.

[vdDMM97] L. van den Dries, A. Macintyre, and D. Marker, Logarithmic-exponential power series, J. London Math. Soc. (2) 56 (1997), no. 3, 417-434.

[vdDMM01] _ Logarithmic-exponential series, Proceedings of the International Conference "Analyse \& Logique" (Mons, 1997), vol. 111, 2001, pp. 61-113. 
[É92] J. Écalle, Introduction aux fonctions analysables et preuve constructive de la conjecture de Dulac, Actualités Mathématiques. [Current Mathematical Topics], Hermann, Paris, 1992.

[Ehr94] P. Ehrlich, All numbers great and small, Real numbers, generalizations of the reals, and theories of continua, Synthese Lib., vol. 242, Kluwer Acad. Publ., Dordrecht, 1994, pp. 239-258.

[Ehr01] P. Ehrlich, Number systems with simplicity hierarchies: a generalization of Conway's theory of surreal numbers, J. Symbolic Logic 66 (2001), no. 3, 1231-1258.

[Ehr12] P. Ehrlich, The absolute arithmetic continuum and the unification of all numbers great and small, Bull. Symbolic Logic 18 (2012), no. 1, 1-45.

[EK] P. Ehrlich and E. Kaplan, Number systems with simplicity hierarchies: a generalization of Conway's theory of surreal numbers II. arXiv:1512.04001 [math.LO].

[FS96] M. Fitting and R. M. Smullyan, Set theory and the continuum problem, Oxford Logic Guides, vol. 34, The Clarendon Press, Oxford University Press, New York, 1996, Oxford Science Publications.

[G16] A. Gehret, Defending the $\lambda$-freedom of $H$-fields. arXiv:1608.00997 [math.LO].

[Gon86] H. Gonshor, An introduction to the theory of surreal numbers, London Mathematical Society Lecture Note Series, vol. 110, Cambridge University Press, Cambridge, 1986.

[Gro97] A. Grothendieck, Esquisse d'un programme. In Geometric Galois actions, 1. London Math. Soc. Lecture Note Ser. 242, Cambridge Univ. Press, Cambridge, 1997, 5-48.

[Hah07] H. Hahn, Über die nichtarchimedischen Grössensystem, Sitzungsberichte der Kaiserlichen Akademie der Wissenschaften, Mathematisch - Naturwissenschaftliche Klasse (Wien) 116 (1907), no. Abteilung IIa, 601-655.

[Har10] G. H. Hardy, Orders of infinity: The 'Infinitärcalül' of Paul du Bois-Reymond. Cambridge Tracts in Mathematics and Mathematical Physics 12, Cambridge University Press, 1910.

[Hau44] F. Hausdorff, Mengenlehre, Dover Publications, New York, N. Y., 1944.

[vdH97] J. van der Hoeven, Asymptotique automatique, Université Paris VII, Paris, 1997, Thèse, Université Paris VII, Paris, 1997, With an introduction and a conclusion in French.

[vdH06] Transseries and real differential algebra, Lecture Notes in Mathematics, vol. 1888, Springer-Verlag, Berlin, 2006.

[Kap42] I. Kaplansky, Maximal fields with valuations. Duke Math. Journal 9 (1942), 303-321.

[Kuh11] F.-V. Kuhlmann, Maps on ultrametric spaces, Hensel's Lemma, and differential equations over valued fields, Communications in Algebra 39:5 (2011), 1730-1776.

[KKS97] F.-V. Kuhlmann, S. Kuhlmann, and S. Shelah, Exponentiation in power series fields Proc. Amer. Math. Soc. 125 (1997), 3177-3183

[Knu74] D. E. Knuth, Surreal numbers, Addison-Wesley Publishing Co., London, 1974.

[Kuh00] S. Kuhlmann, Ordered exponential fields, Fields Institute Monographs, vol. 12, American Mathematical Society, Providence, RI, 2000.

[KM11] S. Kuhlmann and M. Matusinski, Hardy type derivations on fields of exponential logarithmic series., J. Algebra 345 (2011), 171-189.

[KM12] Hardy type derivations in generalized series fields., J. Algebra 351 (2012), 185-203.

[KM15] - The Exponential-Logarithmic Equivalence Classes of Surreal Numbers., Order 32 (2015), 53-68.

[KS05] S. Kuhlmann and S. Shelah $\kappa$-bounded Exponential Logarithmic Power Series Fields Annals for Pure and Applied Logic, 136, 284-296, (2005).

[KT12] S. Kuhlmann and M. Tressl, Comparison of Exponential-Logarithmic and Logarithmic-Exponential series, Math. Logic Quarterly, 58 (2012), 434-448

[MM97] David Marker and Chris Miller, Levelled o-minimal structures, Rev. Mat. Univ. Complut. Madrid 10 (1997), no. Special Issue, suppl., 241-249, Real algebraic and analytic geometry (Segovia, 1995).

[Mat14] M. Matusinski, On generalized series fields and exponential-logarithmic series fields with derivations, Valuation theory in interaction, EMS Ser. Congr. Rep., Eur. Math. Soc., Zürich, 2014, pp. 350-372.

[N49] B. H. Neumann, On ordered division rings. Trans. Amer. Math. Soc. 66 (1949), $202-252$. 
SURREAL NUMBERS WITH DERIVATION, HARDY FIELDS AND TRANSSERIES

[PC83] S. Prieß-Crampe, Angeordnete Strukturen. Gruppen, Körper, projektive Ebenen, Ergebnisse der Mathematik und ihrer Grenzgebiete 98, Springer (1983).

[Res93] J.-P. Ressayre, Integer parts of real closed exponential fields. In Arithmetic, Proof Theory and Computational Complexity (P. Clote and J. Krajicek, eds.), Oxford University Press, 1993, 278-288.

[Rob72] A. Robinson, On the real closure of a Hardy field, Theory of sets and topology (in honour of Felix Hausdorff, 1868-1942), VEB Deutsch. Verlag Wissensch., Berlin, 1972, pp. 427-433.

[Ros81] M. Rosenlicht, On the Value Group of a Differential Valuation II, American Journal of Mathematics 103:5 (1981), 977-996.

[Ros83] _ Hardy fields. J. Math. Anal. Appl. 93 (1983) no. 2, 297-311.

[Ros83] The rank of a Hardy field. Trans. Amer. Math. Soc. 280 (1983) no. 2, 659-671.

[Ros87] M. Rosenlicht, Growth properties of functions in Hardy fields, Trans. Amer. Math. Soc. 299 (1987), no. 1, 261-272. MR MR869411 (88b:12010)

[Ros95] M. Rosenlicht, Asymptotic solutions of $Y^{\prime \prime}=F(x) Y$. J. Math. Anal. Appl. 189 (1995) no. 3, 640-650.

[Sch01] M.C. Schmeling, Corps de transséries, Ph.D. thesis, Université Paris-VII, 2001.

[Sie65] W. Sierpiński, Cardinal and ordinal numbers, Second revised edition. Monografie Matematyczne, Vol. 34, Państowe Wydawnictwo Naukowe, Warsaw, 1965.

[Wil96] A. J. Wilkie, Model completeness results for expansions of the ordered field of real numbers by restricted Pfaffian functions and the exponential function, J. Amer. Math. Soc. 9 (1996), no. 4, 1051-1094.

UNIVERSITY OF LEEDS

E-mail address: v.l.mantova@leeds.ac.uk

UNIVERSITY OF BORDEAUX

E-mail address: mickael.matusinski@math.u-bordeaux.fr 